\title{
Analytische Betrachtung von gewickelten Pressverbänden in Anwendung bei Axialflussmaschinen
}

\author{
Rainer Riegler ${ }^{1} \mathbb{D} \cdot$ Manfred Grafinger $^{1} \mathbb{D}$ \\ Eingegangen: 8. November 2019/ Angenommen: 19. Oktober 2020 / Online publiziert: 6. November 2020 \\ (c) Der/die Autor(en) 2020
}

\section{Zusammenfassung}

Permanenterregte Axialflussmaschinen weisen gegenüber Radialflussmaschinen Vorteile bezüglich der möglichen Abmessungen auf. Bis jetzt wurden vor allem Axialflussmaschinen mit einer vollen Rotorscheibe untersucht. Aufgrund von besseren magnetischen Eigenschaften sind Rotoren aus geschichtetem Elektroblech hergestellt. Radial ausgerichtet führt das zu hohen Kosten, die durch Wicklung des Elektroblechs bei denselben Eigenschaften wesentlich reduziert werden sollen. Die naive numerische Untersuchung mittels FEM scheitert an der großen Anzahl an Kontaktflächen und noch unbekanntem Einfluss der Vorspannung.

Um die mechanische Vorauslegung und Nachrechnung zu ermöglichen werden hier Methoden und Vereinfachungen diskutiert, um Aussagen zur Realisierbarkeit zu erhalten. Es wird das aufgewickelte Blech nicht als archimedische Spirale gerechnet, sondern als Blechringe, die auf die Abtriebswelle aufgezogen werden. Die Abmessungen sind im Vorhinein nur teilweise bekannt und dementsprechend werden mögliche Näherungen angeführt.

Zur Bewertung der Beanspruchung werden vorerst die Vorspannungen bei unendlich dünnem Elektroblech bestimmt und die Anwendbarkeit und Genauigkeit der Gleichungen bei endlich dünnem Elektroblech festgestellt.

Durch diese Vorgehensweise vereinfacht sich die Berechnung erheblich und es kann während der Konstruktion auf die auftretenden Belastungen reagiert werden.

\section{Analytical study of wound fits for the application in axial-flux motors}

\begin{abstract}
Permanent magnet axial flux electric motors are advantageous regarding its dimensions in comparison to radial flux motors in certain applications. Until now, most machines that have been investigated are using full runner discs. Laminated runners provide better properties. Common designs use radially oriented electrical steel sheets. These designs are expensive to manufacture. If those sheets are wound about the rotational axis, the costs are reduced while maintaining the electrical properties.

The direct numerical investigation using a FEM is unfeasible and of low accuracy due to many contact surfaces. Further the influence of the winding preload is not easy to implement.

In order to analyse mechanically, methods and simplifications are discussed here in order to obtain statements on the feasibility. The electrical steel sheets are not modelled as Archimedean spirals, but as several metal rings which are mounted onto the output shaft. The difficulty lies in only a partial knowledge of the geometry before mounting. Accordingly, possible approximations are presented in this paper.

Preload stresses are calculated for infinitely thin steel sheets first and the applicability and accuracy for finitely thin steel sheets are determined.

This procedure simplifies the computation considerably and it can be used to predict the design limits.
\end{abstract}

Rainer Riegler

rainer.riegler@tuwien.ac.at

Manfred Grafinger

manfred.grafinger@tuwien.ac.at
E307-04 - Forschungsbereich Maschinenbauinformatik und Virtuelle Produktentwicklung, TU Wien (TUW), Getreidemarkt 9, 1060 Wien, Österreich 


$\begin{array}{ll}\text { Abkürzungen } & \\ p_{F}(r) & \text { Fugendruck bei Radius [Pa] } \\ \sigma_{m} & \text { Mittlere Vorspannkraft [Pa] } \\ p_{g} & \text { Gesamtfugendruck [Pa] } \\ \sigma_{V}(r) & \text { Radiusabhängige Vorspannung [Pa] } \\ c_{0} & \text { Konstanter Term von Vorspannungspoly- } \\ & \text { nom [Pa] } \\ c_{a} & \text { Term bei } x^{a} \text { in Vorspannungspolynom } \\ & {\left[\frac{P a}{m^{a}}\right]} \\ \sigma_{r}(r) & \text { Radiale Spannung bei Radius [Pa] } \\ \sigma_{t}(r) & \text { Tangentiale Spannung bei Radius [Pa] } \\ r_{W} & \text { Wellenradius [m] } \\ \mathrm{t} & \text { Blechdicke [m] } \\ r_{a} & \text { Untere Integrationsgrenze von Radius [m] } \\ r_{b} & \text { Obere Integrationsgrenze von Radius [m] } \\ \mathrm{Q} & \text { Durchmesser- bzw. Radiusverhältnis [-] } \\ r_{i} & \text { Innenradius vor dem Aufziehen des } \\ u_{A}\left(r_{i}\right) & \text { Blechrings [m] } \\ u_{I}\left(r_{w}\right) & \text { Verschiebung des Innenradius beim Auf- } \\ \mathrm{E} & \text { ziehen des Blechrings [m] } \\ \nu & \text { Verschiebung des Außenradius der Welle } \\ p_{F g}\left(r_{W}\right) & \text { beim Aufziehen des Blechrings [m] } \\ r_{F}\left(r_{W}\right) & \text { Elastizitätsmodul [Pa] } \\ K_{p_{F}} & \text { Querkontraktionszahl [-] } \\ & \text { Fugendruck bei endlich dünnen Blechen } \\ & \text { beim Wellenradius [Pa] } \\ \text { Fugendruck bei unendlich dünnen Ble- } \\ \text { chen beim Wellenradius [Pa] }\end{array}$

\section{Einführung}

Permanenterregte Axialflussmaschinen sind Synchronmaschinen mit magnetischem Fluss in axialer Richtung. Der Rotor konventioneller Axialflussmaschinen wird entweder aus einer Vollscheibe oder aus radial ausgerichteten Elektroblechen hergestellt. Die Verwendung von korrekt ausgerichteten Elektroblechen führt zu geringeren elektrischen Verlusten, allerdings erhöhen diese üblicherweise die Herstellkosten. Um die Vorteile beider Varianten zu kombinieren wurden Rotoren aus gewickeltem Elektroblech entwickelt.

In Abb. 1 ist solch ein Rotor exemplarisch dargestellt, wobei die Bleche übertrieben dick dargestellt wurde, um die Wicklung besser darzustellen.

Bis jetzt wurden solche Maschinen nur elektrisch und thermisch [1], bzw. wurde die Homogenisierung von Elektroblechpaketen, zur Abschätzung der Dämpfung in Rotoren [2], untersucht.
Die direkte Untersuchung des Vorspannungszustands mit der FEM ist nicht praktikabel. Dazu müsste der gesamte Aufwickelvorgang von einem mehr als $100 \mathrm{~m}$ langen Blechstreifen simuliert werden und anschließend der Rotor belastet werden. Mit impliziten Verfahren lässt sich u. a. wegen der großen Anzahl an Kontakten nicht ohne weiteres Konvergenz erreichen. Explizite Verfahren stellen aufgrund der Vielzahl an benötigten Zeitschritten Hindernisse in der Berechnungszeit dar. Dementsprechend ergeben sich daraus auch Probleme in den Zyklenzeiten der Produktentwicklung. Außerdem lassen sich ohne Parameterstudien keine Aussagen darüber treffen, welche Parameter verändert werden müssen. Diese Probleme werden durch eine zumindest teilweise analytische Beschreibung vermieden.

Das Elektroblech wird unter einer bestimmten Vorspannung auf einer Welle aufgewickelt. Durch das Aufwickeln entsteht zwischen den Blechlagen ein Fugendruck, der einerseits von der Vorspannkraft selbst und andererseits von der Anzahl der darüber liegenden Lagen abhängt.

\section{Modellierung und Vereinfachungen}

Hier werden analytische Untersuchungen zur Auslegung und Nachrechnungen vorgestellt. Diese können sowohl zu einer Vorentwurfsrechnung verwendet werden als auch zur etwaigen Vereinfachung einer FEM Rechnung benutzt werden, um zu vermeiden den gesamten Aufwickelvorgang zu simulieren.

\subsection{Voraussetzungen und Einschränkungen}

Das aufgewickelte Blech hat die Form einer archimedischen Spirale. Weil das für E-Maschinen verwendete Elektroblech sehr dünn im Vergleich zum Wickeldurchmesser ist, wird für die Berechnung angenommen, dass Blechringe auf eine Welle aufgezogen werden. Damit die Beanspruchungen ähnlich zur Realität sind, werden die Abmessungen der Ringe so gewählt, dass das Integral der Tangentialspannungen gleich groß der verwendeten Wickelvorspannkraft ist.

Es soll die Blechbreite konstant und gleich der Wellenlänge sein. Die Welle soll als Vollwelle ausgeführt sein. Außerdem sollen die Welle und das Elektroblech dieselben elastischen Materialeigenschaften aufweisen.

Es wird das System außerdem rotationssymmetrisch betrachtet. Das heißt, alle Kräfte und Blechvariationen werden gemittelt betrachtet.

\subsection{Unendlich dünne Bleche}

Zur vernünftigen analytischen Bestimmung der Fugendrücke und der Spannungsverläufe wird die Vorspannkraft auf eine mittlere Vorspannkraft umgerechnet und analog 
Abb. 1 Rotor aus gewickeltem Blech, Blech übertrieben dick dargestellt

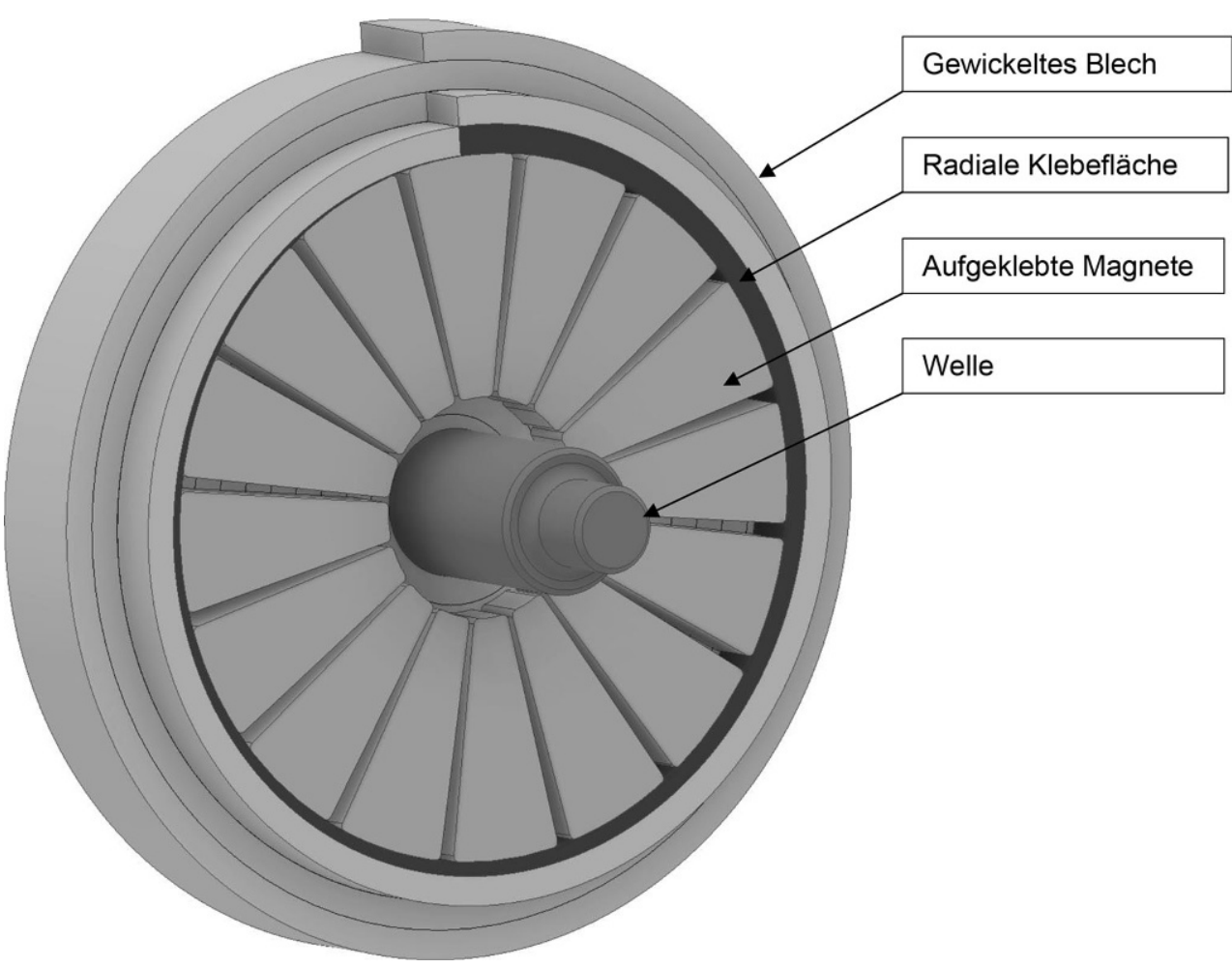

dem Kräftegleichgewicht einer Seilrolle der Fugendruck bestimmt.

$p_{F}(r)=\sigma_{m} \frac{d r}{r}$

Aufgrund dieses Fugendrucks ergibt sich ein Spannungsverlauf im Wellen- und im Ringteil wie bei Zylinderringen unter Innen- bzw. Außendruck. Die Radial- und Tangentialspannungen bei Vollwellen unter Außendruck sind unabhängig vom Radius und sind so groß wie der auftretende Fugendruck [3].

Den Fugendruck an einem bestimmten Radius $r$ ergibt sich damit als Summe der Fugendrücke aller darüberliegenden Ringe, bzw. als das Integral der Fugendrücke von $r$ bis zum Radius des äußersten Ringes R.

$p_{g}=\int_{r}^{\mathrm{R}} p(x)=\int_{r}^{R} \sigma_{V}(x) \frac{d x}{x}$

Unter anderem für Terme von Polynomfunktionen für $\sigma_{V}(r)$ lässt sich der gesamte Fugendruck geschlossen angeben. Der Grad des Terms wird mit a angegeben:

$p_{g}(r)=\int_{r}^{R} c_{a} x^{a} \frac{d x}{x}=\left\{\begin{array}{c}c_{0} \ln \left(\frac{R}{r}\right) \text { wenn } a=0 \\ \frac{c_{a}}{a}\left(R^{a}-r^{a}\right) \text { wenn } a \neq 0\end{array}\right.$
Die wirkende Radialspannung entspricht dem negativen Fugengesamtdruck und die Tangentialspannungen entsprechen der Wickelvorspannung abzüglich dem Fugengesamtdruck.

$\sigma_{r}(r)=-p_{g}(r)$
$\sigma_{t}(r)=\sigma_{V}(r)-p_{g}(r)$

In Abb. 2 sind die Radial- und Tangentialspannungen bei unterschiedlichen Außenradien dargestellt. Der Wickelradius $r$ ist auf den Wellenradius $r_{W}$ normiert.

Die Radial- und Tangentialspannung ist bei unterschiedlichen Außenradien unterschiedlich, weil im Wesentlichen über einen größeren Bereich integriert wird.

In Abb. 2 ist ersichtlich, dass die Tangentialspannungen bei $r / r_{W}=1$ einen Sprung aufweisen. Das liegt daran, dass die Vorspannung nur in den gewickelten Lagen bei $\mathrm{r} / r_{W}>$ 1 auftritt. Der Sprung im Tangentialspannungsverlauf bei den quadratischen Vorspannungen muss nicht zwingender Weise kleiner als bei konstanter Vorspannung sein.

\subsection{Betrachtung endlich dünner Bleche und mögliche Näherungen}

Auch wenn die Blechdicke im Vergleich zum Wickelradius klein ist, muss zum Nachweis, dass die Gleichungen für den Fugendruck eine gute Näherung darstellen der eigentlich vorhandene Spannungszustand ermittelt werden. Dazu wird 
Abb. 2 Vergleich der Spannungsverläufe bei konstanter Vorspannung und quadratischer Verläufe mit $\sigma_{V}(r)=c_{2} x^{2}$ bei unterschiedlichen Außenradien, Spannungen über dem auf den Wellenradius normierten Wickelradius aufgetragen
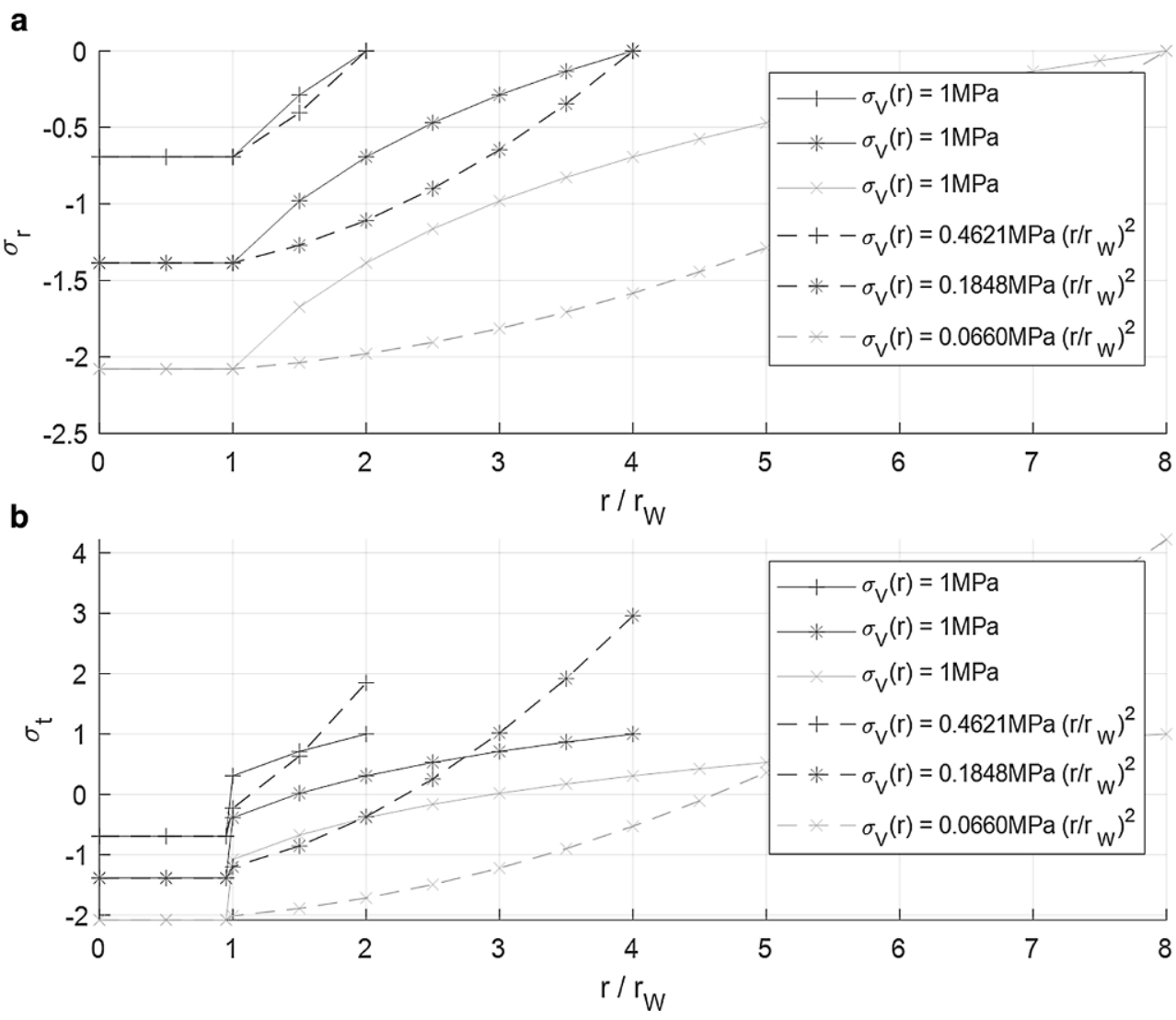

das aufgewickelte Blech wie ein Hohlzylinder behandelt. Problematisch beim Bestimmen des Spannungszustandes ist, dass zwar die Dicke des Blechs im Ausgangszustand bekannt ist, aber die Ringzugkräfte erst im aufgezogenen Zustand.

\subsubsection{Bestimmung des Fugendrucks (abhängig von $r_{i}$ )}

Die Tangentialspannung lässt sich wie folgt darstellen [3], wobei $r_{a}$ der Innenradius des aufzuziehenden Blechringes ist, $r_{b}$ der Außenradius und $\mathrm{t}$ die Blechstärke:

$$
\begin{gathered}
\sigma_{t}(r)=C_{1}-\frac{C_{2}}{r^{2}}=\frac{p_{F}}{1-Q^{2}} \cdot\left(Q^{2}+\frac{r_{a}^{2}}{r}\right) \\
=p_{F} \cdot \frac{Q^{2}}{1-Q^{2}} \cdot\left(1+\frac{r_{b}{ }^{2}}{r^{2}}\right) \\
\mathrm{Q}=\frac{r_{b}}{r_{a}}
\end{gathered}
$$

Es soll nun die Tangentialspannung über den Radius aufintegriert werden um die im Ring wirkende Zugkraft zu erhalten. Diese wird in weiterer Folge mit der Zugkraft durch die Wickelvorspannung gleichgesetzt. Damit wird eine Be- ziehung zwischen dem Fugendruck und der Wickelvorspannung erhalten.

$$
\begin{aligned}
\int_{r_{a}}^{r_{b}} \sigma_{t}(r) d r= & \int_{r_{a}}^{r_{b}} p_{F} \cdot \frac{Q^{2}}{1-Q^{2}} \cdot\left(1+\frac{r_{b}^{2}}{r^{2}}\right) d r=p_{F} \frac{Q^{2}}{1-Q^{2}} \\
& \left(\frac{r_{b}^{2}}{r_{a}}-r_{a}\right)=p_{F} \frac{Q^{2}}{1-Q^{2}} r_{a}\left(Q^{2}-1\right)= \\
& -p_{F} \frac{r_{b}^{2}}{r_{a}}=-p_{F} \frac{\left(r_{a}+t\right)^{2}}{r_{a}}=-\sigma_{V} \cdot t
\end{aligned}
$$

Nach Umformung auf $p_{F}$ und ersetzen der Bezeichnung für den Innenradius $r_{a}$ zu $r_{i}$ ergibt sich:

$p_{F}=\frac{\sigma_{V}}{\frac{r_{i}}{t}+2+\frac{t}{r_{i}}}$

Dabei ist $r_{i}$ noch unbekannt.

\subsubsection{Bestimmung des nötigen Innendurchmessers beim Aufziehen von Blechringen}

Der Innendurchmesser des Blechringes und der Außendurchmesser des Innenteils soll nach der Beanspruchung mit dem vorhin bestimmten Fugendruck den gleichen Durchmesser aufweisen. Die Beanspruchung bewirkt De- 
Abb. 3 Spannungsverläufe bei endlicher Blechdicke
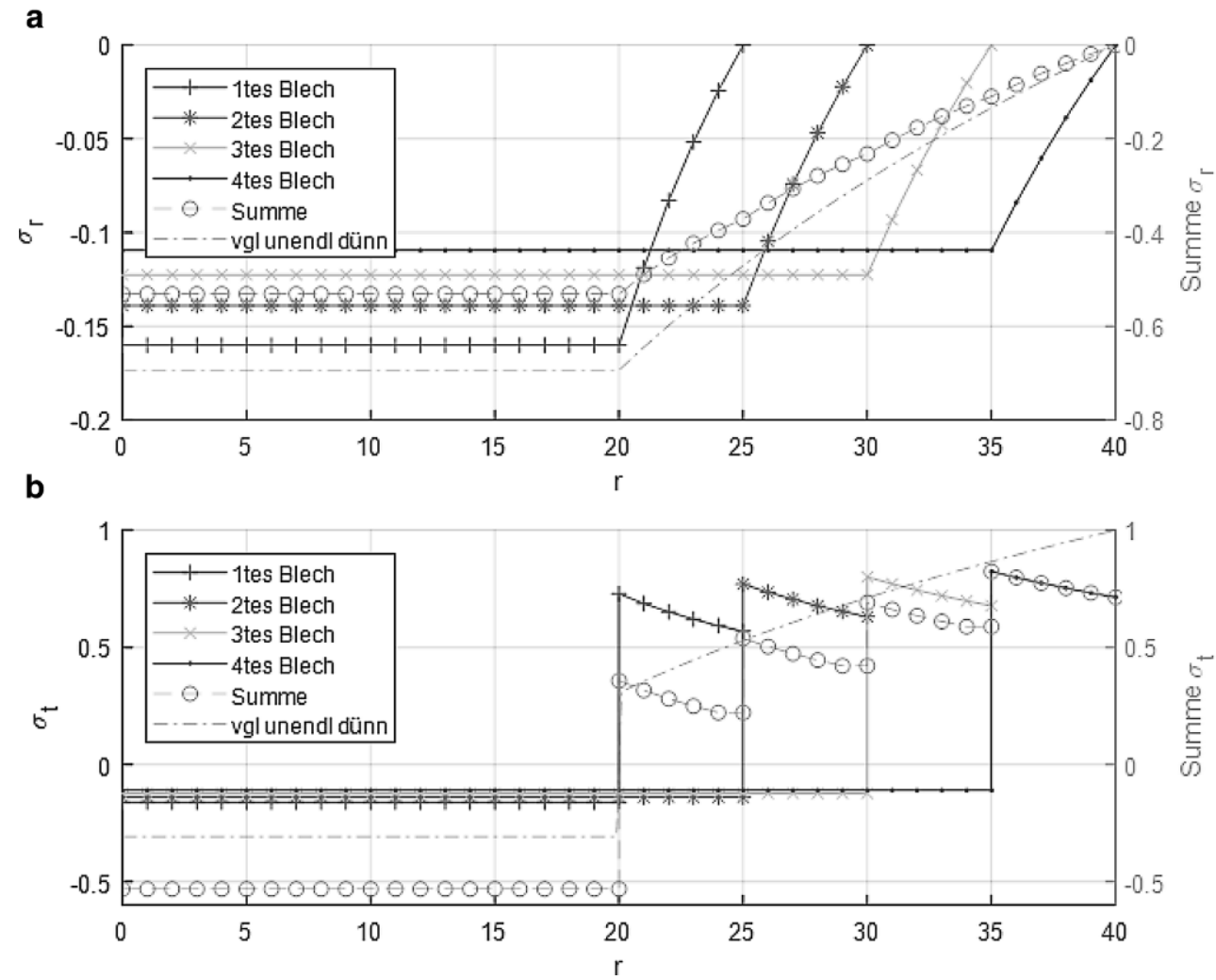

formationen des Blechringes $\left(u_{A}\left(r_{i}\right)\right)$ und der Welle $\left(u_{I}\left(r_{w}\right)\right)$.

$r_{i}+u_{A}\left(r_{i}\right)=r_{w}+u_{I}\left(r_{w}\right)$

Die Deformationen lassen sich wie die Spannungen mit dem Fugendruck bestimmen [3]:

$$
\begin{aligned}
u_{A}\left(r_{i}\right)= & -p_{F} r_{i} \cdot \frac{1}{E} \cdot\left(\frac{1+Q_{A}^{2}}{1-Q_{A}^{2}}+\nu\right)=p_{F} r_{i} \cdot \frac{1}{E} \\
& \cdot\left(\frac{2 r_{i}^{2}+2 r_{i} t+t^{2}}{2 r_{i} t+t^{2}}+v\right) \\
u_{I}\left(r_{w}\right)= & -p_{F} r_{W} \cdot \frac{1}{E}(1-v)
\end{aligned}
$$

Für eine Vollwelle als Innenteil und gleiche elastische Materialparameter ergibt sich diese Beziehung zur Ermittlung von $r_{i}$ :

$$
\begin{aligned}
& r_{i}+p_{F} r_{i} \cdot \frac{1}{E} \cdot\left(\frac{2 r_{i}^{2}+2 r_{i} t+t^{2}}{2 r_{i} t+t^{2}}+\nu\right)=r_{w}-p_{F} r_{W} \cdot \frac{1}{E}(1-v) \\
& r_{w}=r_{i} \frac{\left(\frac{r_{i}}{t}+2+\frac{t}{r_{i}}\right)+\frac{\sigma_{V}}{E}\left(\frac{2 r_{i}^{2}+2 r_{i} t+t^{2}}{2 r_{i} t+t^{2}}+\nu\right)}{\left(\frac{r_{i}}{t}+2+\frac{t}{r_{i}}\right)-\frac{\sigma_{V}}{E}(1-\nu)}
\end{aligned}
$$

Diese Gleichung kann numerisch für $r_{i}$ gelöst werden.
Aufgrund der geringen Blechdicke im Vergleich zum Wickelradius und den Eigenschaften von den meisten technischen Werkstoffen $\left(\sigma_{F} / \mathrm{E}\right.$ für Metalle liegt im Bereich von etwa 1 bis $0,01 \%$ [4]) lassen sich folgende Näherungen definieren:

$$
\begin{aligned}
& \frac{2 r_{i}^{2}+2 r_{i} t+t^{2}}{2 r_{i} t+t^{2}}=\frac{2 r_{i}^{2} \cdot\left(1+\frac{t}{r_{i}}+\frac{t^{2}}{2 r_{i}^{2}}\right)}{2 r_{i} t \cdot\left(1+\frac{t}{2 r_{i}}\right)} \approx \frac{r_{i}}{t} \\
& \left(\frac{r_{i}}{t}+2+\frac{t}{r_{i}}\right)=\frac{r_{i}}{t}\left(1+2 \frac{t}{r_{i}}+\frac{t^{2}}{r_{i}^{2}}\right) \approx \frac{r_{i}}{t} \\
& \frac{t}{r_{i}} \approx 0,1 \ll \frac{r_{i}}{t}, \frac{\sigma_{V}}{E}<\frac{\sigma_{F}}{E} \ll 1
\end{aligned}
$$

Und es ergibt sich somit, dass als gute Näherung für $r_{\mathrm{i}}$ der Radius des Innenteils verwendet werden kann:

$$
r_{w} \approx r_{i} \frac{\frac{r_{i}}{t} \cdot\left(1+\frac{\sigma_{V}}{E}\left(1+\frac{t}{r_{i}} \cdot \nu\right)\right)}{\frac{r_{i}}{t} \cdot\left(1-\frac{\mathrm{t}}{r_{i}} \frac{\sigma_{V}}{E}(1-\nu)\right)} \approx r_{i} \cdot\left(1+\frac{\sigma_{V}}{E}\right) \approx r_{i}
$$

\subsubsection{Bestimmung des Spannungszustandes}

Für Vollwellen ist der gesamte Fugendruck in einer Schichtdicke die Summe der darüberliegenden Fugendrücke, da in 
Abb. 4 Korrekturfaktor bei konstanter Vorspannung

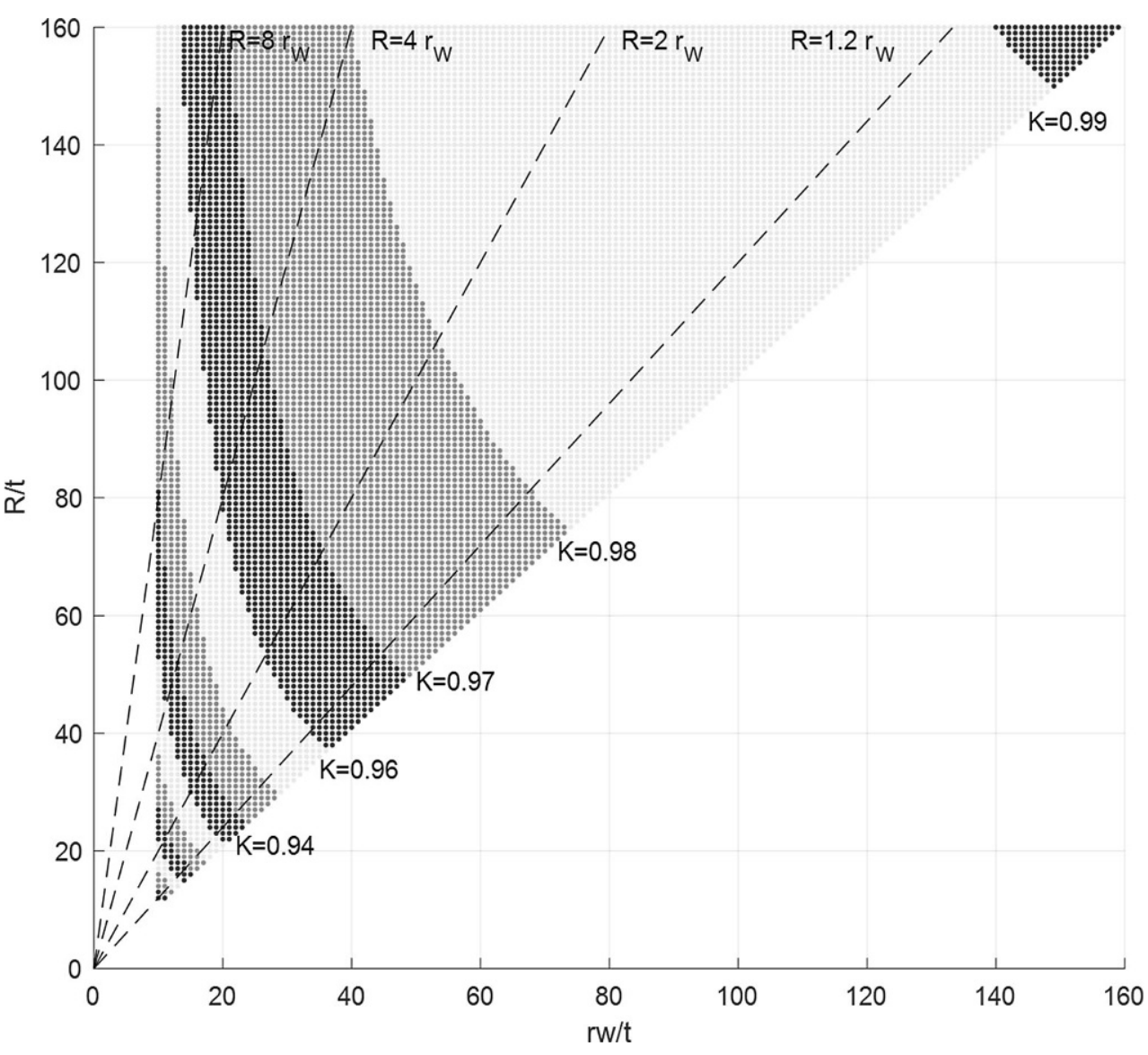

einem Querpressverband mit einer Vollwelle die Radialspannungen im Innenteil konstant sind [5].

Das Elektroblech wird unter Vorspannung aufgewickelt. Diese Vorspannung bleibt einerseits als Tangentialspannung nach dem Wickelvorgang im Blech bestehen und andererseits erzeugt der Fugendruck der darüberliegenden Schichten eine Tangentialspannung. Diese Tangentialspannungen werden überlagert Damit ergibt sich für die wirkende Tangentialspannung einer Schicht, dass von der Vorspannung, der in der Schicht wirkende Gesamtfugendruck abgezogen wird.

In Abb. 3 ist ersichtlich, dass die Kurvenform der Radialspannung über dem Radius selbst bei Verwendung von vergleichsweise dicken Blechen noch gut durch die unter 2.2 für unendlich dünne Bleche vorgestellte analytische Lösung bestimmt werden kann. Durch die verwendeten Näherungen kommt es allerdings für dickere Bleche zu signifikanten Abweichungen, die Korrekturfaktoren notwendig machen.

\subsubsection{Korrekturfaktor des Fugendrucks}

Um die Näherungsformeln benutzen zu können, bzw. die Verwendbarkeit der Näherungsformel für den Fugendruck abschätzen zu können wird hier ein Korrekturfaktor ermit- telt. Der Korrekturfaktor ist das Verhältnis des Fugendrucks beim Wellenradius bei endlicher Blechdicke zum Fugendruck bei unendlich dünner Blechdicke.

$K=\frac{p_{F g}\left(r_{W}\right)}{p_{g}\left(r_{W}\right)}$

Der gesamte Fugendruck am Wellenradius lässt sich als Summe der Fugendrücke aller Schichten ausdrücken. Unter Verwendung der Formel aus 2.3.1 ergibt sich:

$$
\begin{aligned}
p_{F g}\left(r_{W}\right)= & \sum_{i=0}^{n} \frac{\sigma_{V}\left(r_{i}\right)}{\frac{r_{i}}{t}+2+\frac{t}{r_{i}}}=\sum_{i=0}^{n} \frac{\sigma_{V}\left(r_{W}+i \cdot t\right)}{\frac{r_{W}+i \cdot t}{t}+2+\frac{t}{r_{W}+i \cdot t}} \\
& =\sum_{i=0}^{\left\lfloor\frac{R}{t}-\left(\frac{r_{W}}{t}+1\right)\right\rfloor} \sigma_{V}\left(r_{W}+i \cdot t\right) \\
& \left(\frac{1}{\left(\frac{r_{W}}{t}+1\right)+i} \cdot\left(1-\frac{1}{\left(\frac{r_{W}}{t}+1\right)+i}\right)\right)
\end{aligned}
$$

Für diesen Ausdruck lässt sich keine allgemein gültige Formel angeben. Deshalb wurde hier für einen konstanten Vorspannungsverlauf der Korrekturfaktor ausgewertet.

In Abb. 4 ist der Korrekturfaktor $\mathrm{K}$ als Isoplot über $\mathrm{R} / \mathrm{t}$ und $r_{W} / t$ dargestellt. Es ist ersichtlich, dass bei geringer 
Abb. 5 Korrekturfaktor bei quadratischer Vorspannung

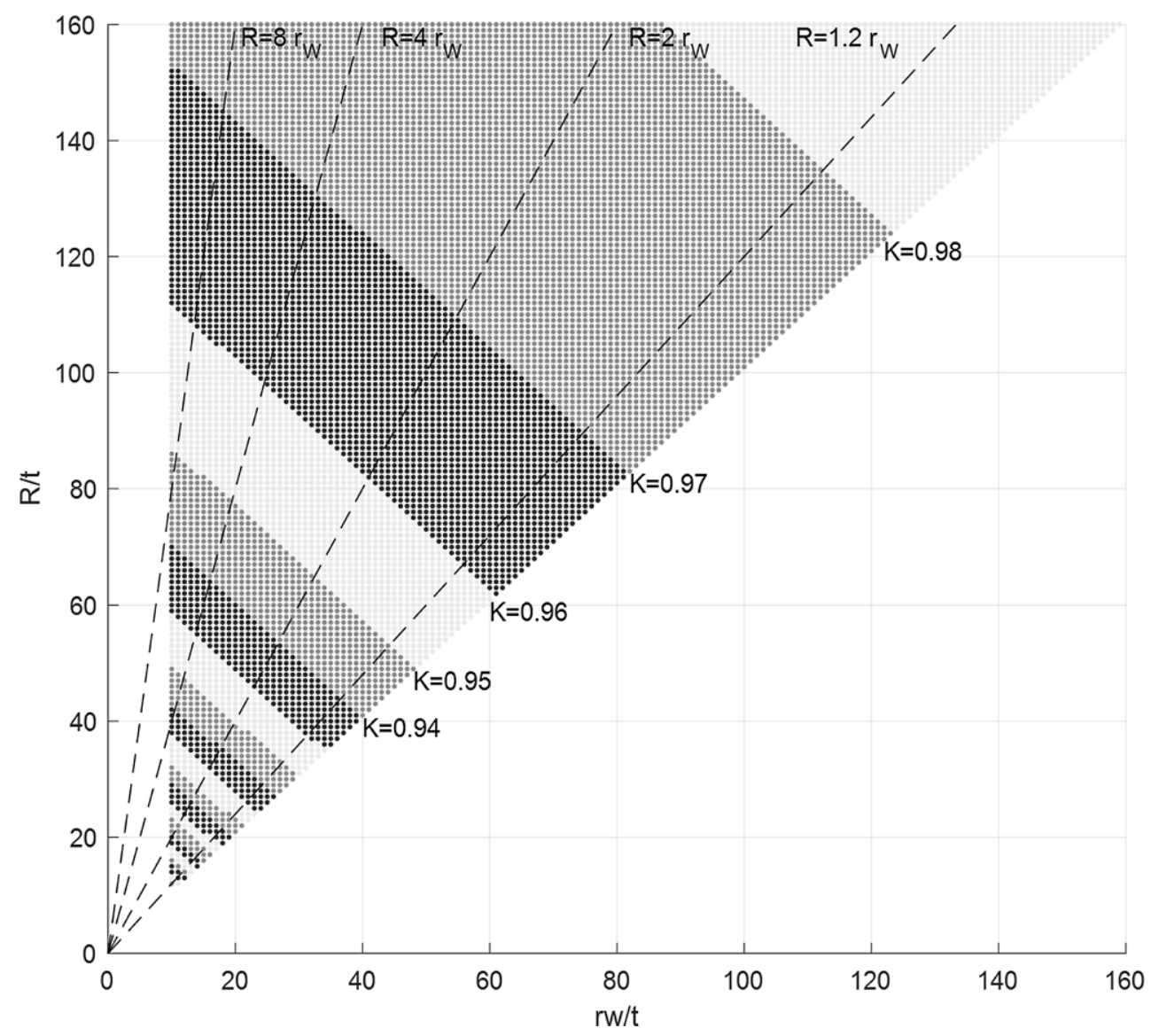

werdender Blechdicke die Genauigkeit der Näherung steigt. Weiters steigt die Genauigkeit je größer der Wellenradius im Verhältnis zum Außenradius ist. Weil der Wellenradius stets kleiner als der Außenradius ist, ist nur die obere Hälfte des Diagramms belegt.

Für den Korrekturfaktor bei quadratischer Vorspannung sind wie in Abb. 5 die gleichen Aussagen gültig. Außerdem ist mit geringerer Genauigkeit, im Vergleich zur konstanten Vorspannung, der Näherung zu rechnen.

\subsection{Vergleich der Varianten konstante Vorspannung und quadratischer Vorspannungsverlauf}

Hier werden die bisherigen Ergebnisse für unterschiedliche Blechdicken verifiziert. Dabei sind die Blechdicken auf den Außenradius normalisiert. $\mathrm{t} / R=0$ repräsentiert dabei die in 2.2 berechneten Ergebnisse. Abb. 6 und 7 zeigen jeweils die Ergebnisse für einen konstanten bzw. einen mit $r$ quadratisch ansteigenden Vorspannungsverlauf. Für die anderen Kurven wurden die Ergebnisse nach 2.3 ermittelt, wobei die Näherung $r_{F} \approx r_{W}$ aus 2.3 .2 benutzt wurde. Die Vorspannung wurde bei $\mathrm{r}=R$ auf 1 normiert und der Fugengesamtdruck bei $\mathrm{r}=r_{W}$ auf 1 normiert.
Analytisch lässt sich der Fugendruck nicht für $\mathrm{t} / R=0$ auswerten, bzw. ist 0 , wenn mit einer Vorspannung und nicht mit einer Vorspannkraft gerechnet wird.

Die Ergebnisse für geringe Blechdicken passen sehr gut zu den analytischen Lösungen und gehen für $\lim _{t \rightarrow 0}$ für die zwei gewählten Vorspannungsverläufe in die analytische Lösung über.

\section{Zusammenfassung und Ausblick}

In dieser Arbeit wurde eine vereinfachte Methode zur Nachrechnung von gewickelten Pressverbänden entwickelt. Einerseits lässt sich für ausreichend dünnes Blech mit guter Näherung ein von der Vorspannung abhängiger Radialspannungs- und Druckverlauf angeben und andererseits kann der Einfluss der Blechdicke berücksichtig werden. Der Fehler, der durch die Verwendung der hier vorgestellten Näherungen für unendlich dünne Bleche entsteht, kann mit den Diagrammen abgeschätzt, oder für beliebige Vorspannungsverläufe berechnet werden.

Die hier gefundenen Ergebnisse gelten nur für Verbände konstanter Breite, können aber bereits zur Vorhersage von Belastungsgrenzen benutzt werden. Außerdem dient die- 
Abb. 6 Vergleich des Fugendrucks bei unterschiedlicher Blechdicke für konstante Vorspannung
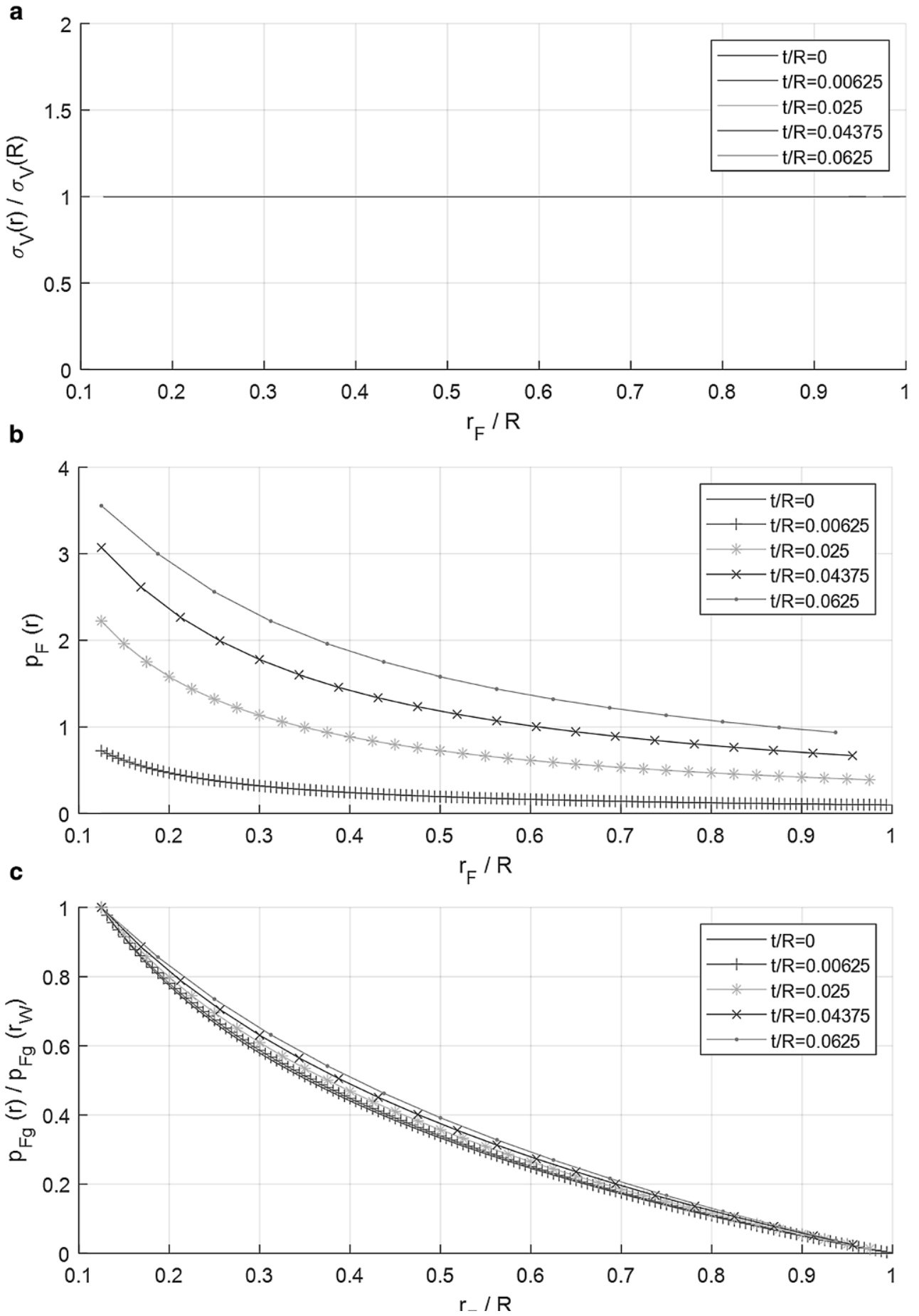
Abb. 7 Vergleich des Fugendrucks bei unterschiedlicher Blechdicke für quadratischen Vorspannungsverlauf
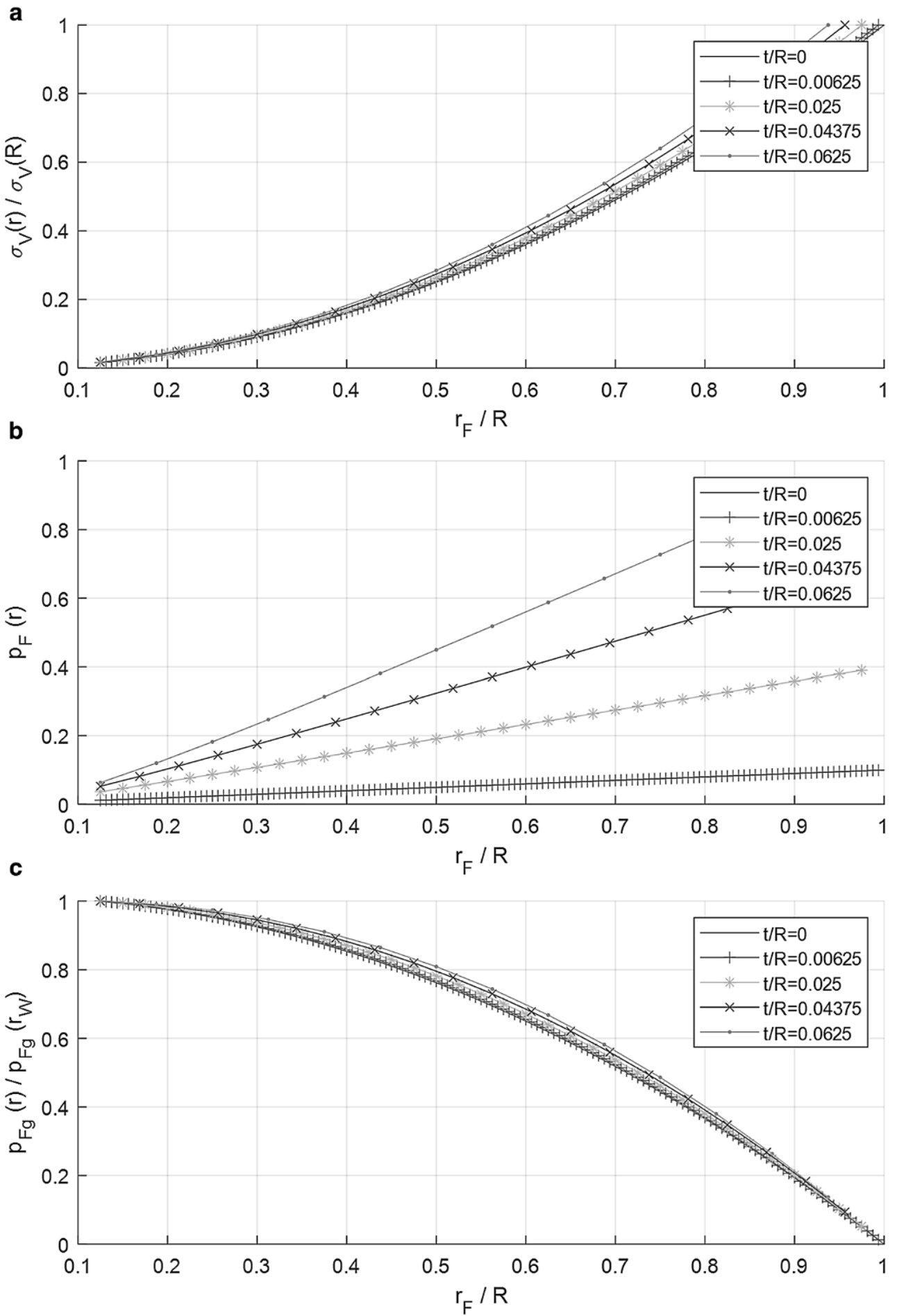
ses Paper als Basis zur Berechnung der Belastungen durch Anbauten, wie die aufgeklebten Magnete. Außerdem beeinflussen die Klebeflächen der Magnete durch die im Einsatz entstehenden Fliehkräfte die zu übertragenden Beanspruchungen.

Auftretende Tangentialspannungen sind in dieser Arbeit unberücksichtigt geblieben und müssen zur Berechnung der erwarteten Einsatzdauer der Maschine bestimmt werden. Unter anderem auch mit Berücksichtigung von möglichen Kerbspannungen. Diesbezüglich werden FEM Untersuchungen unter Verwendung der hier ermittelten Spannungsverhältnisse durchgeführt werden. Außerdem werden die Ergebnisse der Belastungsgrenzen mit Prüfstandsversuchen an einer realen Axialflussmaschine mit gewickeltem Blechrotor verifiziert werden.

Funding Open access funding provided by TU Wien (TUW).

Interessenkonflikt R. Riegler und M. Grafinger geben an, dass kein Interessenkonflikt besteht.

Open Access Dieser Artikel wird unter der Creative Commons Namensnennung 4.0 International Lizenz veröffentlicht, welche die Nutzung, Vervielfältigung, Bearbeitung, Verbreitung und Wiedergabe in jeglichem Medium und Format erlaubt, sofern Sie den/die ursprünglichen Autor(en) und die Quelle ordnungsgemäß nennen, einen Link zur Creative Commons Lizenz beifügen und angeben, ob Änderungen vorgenommen wurden.
Die in diesem Artikel enthaltenen Bilder und sonstiges Drittmaterial unterliegen ebenfalls der genannten Creative Commons Lizenz, sofern sich aus der Abbildungslegende nichts anderes ergibt. Sofern das betreffende Material nicht unter der genannten Creative Commons Lizenz steht und die betreffende Handlung nicht nach gesetzlichen Vorschriften erlaubt ist, ist für die oben aufgeführten Weiterverwendungen des Materials die Einwilligung des jeweiligen Rechteinhabers einzuholen.

Weitere Details zur Lizenz entnehmen Sie bitte der Lizenzinformation auf http://creativecommons.org/licenses/by/4.0/deed.de.

\section{Literatur}

1. Odvářka E (2010) Thermal modeeling of water-cooled axial-flux permanent magnet machine. In: 5th IET International Conference on Power Electronics, Machines and Drives Brighton, UK

2. Luchscheider V (2016) Experimentelle und numerische Identifikation eines homogenisierten Materialmodells für Blechpakete elektrischer Maschinen. Schriftenreihe Technische Mechanik. Erlangen Univ. Erlangen-Nürnberg Lehrstuhl für Techn. Mechanik, Erlangen-Nürnberg

3. Böge A, Böge W, Böge G, Weißbach W (2015) Formeln und Tabellen Maschinenbau, für Studium und Praxis. Springer Vieweg, Wolfenbüttel, S 258

4. Ashby M (2010) Materials selection in mechanical design. Butterworth-Heinemann, Amsterdam, Boston

5. Wittel H, Muhs D, Jannasch D, Voßiek J (2011) Roloff/Matek Maschinenelemente: Normung, Berechnung, Gestaltung. Vieweg+Teubner, Wiesbaden 